\title{
Robust Dual-Band MWIR/LWIR Infrared Target Tracking
}

\author{
Chuong T. Nguyen ${ }^{\dagger}$, Joseph P. Havlicek ${ }^{\dagger}$, Guoliang Fan ${ }^{\ddagger}$, John T. Caulfield ${ }^{\S}$, and Marios S. Pattichis ${ }^{\dagger \dagger}$ \\ ${ }^{\dagger}$ School of Electrical and Computer Engineering, University of Oklahoma, Norman, OK, USA \\ ${ }^{\ddagger}$ School of Electrical and Computer Engineering, Oklahoma State University, Stillwater, OK, USA \\ $\S$ Cyan Systems Inc., Santa Barbara, CA, USA \\ ${ }^{\dagger}$ Dept. Electrical and Computer Engineering, University of New Mexico, Albuquerque, NM, USA
}

\begin{abstract}
We introduce an SIR particle filter for tracking civilian targets including vehicles and pedestrians in dual-band midwave/longwave infrared imagery as well as a novel dualband track consistency check for triggering appearance model updates. Because of the paucity of available dual-band data, we constructed a custom sensor to acquire the test sequences. The proposed algorithm is robust against magnification changes, aspect changes, and clutter and successfully tracked all 17 cases tested, including two partial occlusions. Future work is needed to comprehensively evaluate performance of the algorithm against state-of-the-art video trackers, especially considering the relatively small number of previous dual-band tracking results that have appeared.
\end{abstract}

Keywords-dual-band infrared, sensor fusion, target detection and tracking

\section{INTRODUCTION}

Dual-band infrared (IR) focal plane array (FPA) detectors capable of imaging simultaneously in two wavebands have matured dramatically in the last decade [1]-[5]. Because the thermal signatures of objects and backgrounds are wavelength dependent, this technology can in theory be used to improve target detection, tracking, and clutter rejection performance in a variety of important applications [6]-[8]. For example, dual-band sensors operating in the shortwave IR (SWIR) and midwave IR (MWIR) bands as well as in the MWIR and longwave IR (LWIR) bands have been used in surface-to-air missile seekers to reject countermeasures such as flares [9], [10]. MWIR/LWIR sensors are currently used for shipboard IR search and track (IRST) [11], [12] and MWIR/MWIR sensors have been used to prevent false alarms in aircraft missile warning receivers [13]-[15].

In several countries, Army, Navy, and Air Force investments have been significant in the development of dual-band sensors for the 8-12 $\mu \mathrm{m}$ LWIR band and 3-5 $\mu \mathrm{m}$ MWIR band. These bands are characterized by several important differences. Hot objects such as exhaust ports and engine plumes are more pronounced in the MWIR [7], [10], [16], while airframes, fuselages, and missile hardbodies are more pronounced in the LWIR [7], [10]. Water vapor absorption dominates in the LWIR while carbon dioxide absorption dominates in the

This work was supported in part by the U.S. Army Research Laboratory and the U.S. Army Research Office under grant W911NF-08-1-0293.
MWIR [16]. Solar reflections are significant in the MWIR but suppressed in the LWIR [6], [16]. Because of the greater effects of aerosols in the MWIR, LWIR sensors generally perform better in the presence of haze and certain types of smoke [16]. Hence, a dual-band MWIR/LWIR sensor is expected to be capable of providing improved situational awareness across a wide range of ambient conditions.

By displaying recorded MWIR and LWIR video sequences of targets and backgrounds under various ambient conditions to a human observer, psychophysical tests have been conducted to determine if one of the bands provides inherently superior object detection and false alarm rejection performance [17], [18]. In most cases, the results have not been strongly conclusive and similar performance has been obtained with both bands. The closely related problem of how to fuse the two bands for display to a human observer has been extensively studied [6], [19]-[23].

Comparatively much less work has been devoted to the study of algorithms for dual-band MWIR/LWIR automatic target recognition (ATR), detection, and tracking where the main intended receiver is a machine as opposed to a human. Several authors have considered automated MWIR/LWIR fusion for detection and tracking of small and point targets in IRST applications [11], [12], [24]-[26]. For extended targets, Dainty, et al., integrated a correlator and Kalman filter with an embedded neural network in the tracking loop to track ground vehicles with a dual-band MWIR/LWIR FLIR sensor [27]. They demonstrated a slight improvement in tracking performance by track-level fusion of the two bands relative to tracking either band independently. Using opponent color interaction and motivated by visible/IR fusion in reptiles, Fay, et al., trained fuzzy ARTMAP networks to recognize a variety of targets in dual-band SWIR/LWIR and MWIR/LWIR imagery [28]. The system achieved a high probability of detection with low false alarm rates against small boats in a littoral IRST application. Chan and Nasrabadi used an eigenspace separation transform or PCA for dimensionality reduction and trained a multilayer perceptron to recognize extended targets in dual-band MWIR/LWIR imagery [29], [30]. Compared to single-band processing, they found that both pixel-level and feature-level dual-band fusion could significantly improve the recognition rate while simultaneously reducing false alarms. 


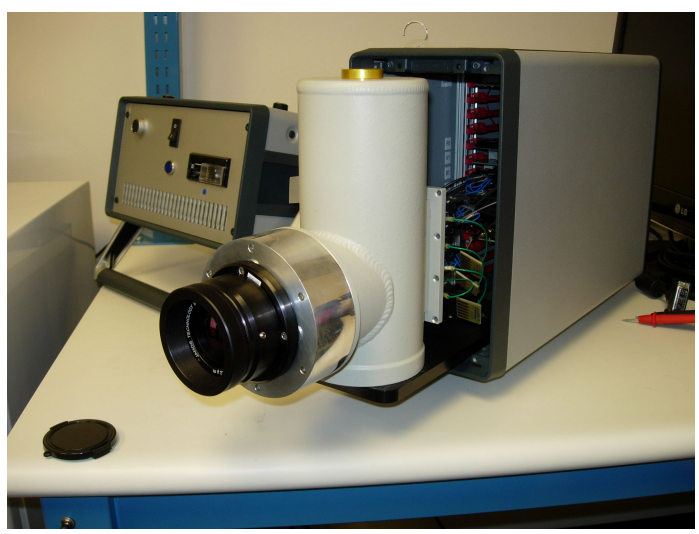

Fig. 1. Dual-band MWIR/LWIR project sensor.

In this paper, we evaluate the performance of a dual-band MWIR/LWIR SIR particle filter with pixel-level fusion for tracking a variety of civilian targets using a simple templatebased appearance model. By also fusing track-level midwave and longwave information, we introduce a powerful new dualband template update policy that prevents track losses and generally improves tracking accuracy relative to a single-band tracker operating in either band alone without updates.

\section{MWIR/LWIR SENSOR AND DATA ACQUISITION}

Due to the profound paucity of MWIR/LWIR target and background signature data in the public domain [31], we constructed a new sensor specifically for the project. The FPA is a $320 \times 240$ MWIR/LWIR QWIP array by QmagiQ Corp., Nashua, NH. As can be seen in Fig. 2, there is a swath of approximately $70 \times 140$ dead pixels in the midwave array; while undesirable, this made it possible to obtain the FPA within the project budget. The FPA was integrated with a DWR-LN2 dewar and CamIRa camera head by SE-IR, Goleta, CA, readout electronics by FLIR Systems, Santa Barbara, CA, and an f/2.3 50mm lens by Janos Technology, Keene, NH, as shown in Fig. 1. With vacuum pumping of the dewar, this sensor achieves an NEDT $<50 \mathrm{mK}$ in both bands at $68 \mathrm{~K}$.

In field tests during August, 2010, and June, 2012, we acquired 48 dual-band sequences depicting hundreds of civilian targets including pedestrians, bicycles, motorcycles, automobiles, trucks, SUV's, buses, RV's, and aircraft. From among these, 17 targets were extracted for initial testing as described in Table I and ground truth was manually compiled in both bands. Sample frame pairs for four of these targets are shown in Fig. 2 with ground truth bounding boxes overlayed.

\section{TRACK FILTER FORMULATION}

We assume a standard constant velocity target model with white noise acceleration. The target state vector is given by

$$
\mathbf{x}_{k}=\left[\begin{array}{llllll}
x_{1, k} & \dot{x}_{1, k} & \delta_{1, k} & x_{2, k} & \dot{x}_{2, k} & \delta_{2, k}
\end{array}\right]^{T},
$$

where $x_{1, k}$ and $x_{2, k}$ are the horizontal and vertical coordinates of the target centroid, $\dot{x}_{1, k}$ and $\dot{x}_{2, k}$ are the horizontal and vertical components of velocity, and $\delta_{1, k}$ and $\delta_{2, k}$ are the
TABLE I

DESCRIPTIONS OF THE 17 TEST TARGETS.

\begin{tabular}{|c|r|l|l|}
\hline Target & $\begin{array}{r}\text { Length } \\
\text { Name }\end{array}$ & $\begin{array}{c}\text { Day / } \\
\text { (frames) }\end{array}$ & \multicolumn{1}{|c|}{$\begin{array}{c}\text { Night } \\
\text { Target } \\
\text { Description }\end{array}$} \\
\hline \hline BC1 & 180 & Day & car receding \\
BC2 & 300 & Day & pickup approaching \\
BC3 & 115 & Day & suv approaching \\
BC4 & 160 & Day & van approaching \\
BC5 & 275 & Day & minivan receding \\
BC6 & 130 & Day & car receding \\
BC7 & 380 & Day & suv receding \\
\hline SA1 & 72 & Day & jet taking off \\
SA2 & 200 & Day & prop plane in flight \\
\hline SB1 & 80 & Day & bicyclist \\
SB2 & 89 & Day & minivan approaching \\
SB3 & 178 & Day & car approaching \\
SB4 & 213 & Day & bicyclist \\
\hline VN1 & 200 & Night & meandering pedestrian \\
VN2 & 190 & Night & suv turning \\
VN3 & 240 & Night & suv approaching \\
VN4 & 300 & Night & pedestrian approaching \\
\hline
\end{tabular}

horizontal and vertical size of the target in pixels. The state update is given by

$$
\mathbf{x}_{k+1}=\mathbf{A} \mathbf{x}_{k}+\mathbf{v}_{k}
$$

where $\mathbf{v}_{k}$ is assumed zero mean and white and $\mathbf{A}=$ $\operatorname{diag}(\mathbf{F} \mathbf{F})$ with

$$
\mathbf{F}=\left[\begin{array}{ccc}
1 & \Delta & 0 \\
0 & 1 & 0 \\
0 & 0 & (1+\gamma)
\end{array}\right]
$$

$\Delta$ is the frame time, and $\gamma$ is a uniform ternary random variable that takes values $\pm \eta$ and zero, each with probability $1 / 3$, to model target magnification changes. For the test sequences in Table I, we used $\eta=0.1$. The target appearance is modeled by a global template $\mathbf{T}$. The observation model is

$$
\mathbf{z}_{k}=H\left(\mathbf{x}_{k}, \mathbf{T}\right)+\mathbf{n}_{k},
$$

where $\mathbf{n}_{k}$ is zero mean additive white noise uncorrelated with $\mathbf{v}_{k}$ and $H$ is a function that resizes $\mathbf{T}$ to an extent of $\delta_{1, k} \times$ $\delta_{2, k}$ pixels by bicubic interpolation.

For tracking, we implemented a new dual-band SIR particle filter (DBSIR) to process dual-band imagery obtained by stacking the incoming midwave and longwave frames. As a baseline for comparison, we also implemented standard SIR particle filters [32] in the longwave band alone (LWSIR) and in the midwave band alone (MWSIR). For each track filter, a template $\mathbf{T}$ was extracted from the initial frame using ground truth. The dual-band template was constructed by stacking the 
individual longwave and midwave templates. The likelihood function and weight update are given by

$$
w_{k}^{i} \propto p\left(\mathbf{z}_{k} \mid \mathbf{x}_{k}^{i}\right)=e^{-\left(1-\rho_{k}^{i}\right)},
$$

where $\rho_{k}^{i}$ denotes the normalized cross correlation between the target appearance hypothesized by the particle $\left\{\mathbf{x}_{k}^{i}, w_{k}^{i}\right\}$ using (4) and a tile of size $\delta_{1, k}^{i} \times \delta_{2, k}^{i}$ pixels cut from around the predicted location $\left[x_{1, k}^{i} x_{2, k}^{i}\right]^{T}$ in the current frame. For the two single-band algorithms LWSIR and MWSIR, the NCC calculation was carried out over the longwave or midwave band only, while the NCC calculation for the twocolor DBSIR algorithm was carried out jointly over the stacked LWIR/MWIR template and observation.

Template updates were not performed by the independent LWSIR and MWSIR trackers. For the DBSIR tracker, a second pair of LWSIR and MWSIR filters were implemented and run in parallel with the DBSIR filter to provide a dual-band track consistency check for performing appearance model updates. What we have observed with these data sequences is that target signature evolution tends to cause a failure in one band before the other. Even when both fail simultaneously, it seems to be rare that the failure modes are similar. Thus, with dualband fusion an impending track loss can often be detected by thresholding the distance between the LWSIR and MWSIR track centroids. Hence, we initiated a template refresh of all three particle filters in the dual-band algorithm from the last reliably tracked frame any time the LWSIR and MWSIR track centroids diverged by more than two pixels.

Thus, the dual-band DBSIR algorithm utilized two-color information in two ways: first, to implement an improved NCC calculation $\rho_{k}^{i}$ in (5) by jointly fusing both bands at the pixel level, and second to implement a dual-band consistency check at the track level for triggering target appearance model updates.

\section{Results}

RMS tracking errors for all three algorithms against the 17 test targets averaged across 40 runs each are given in Table II. Examples are shown in Figs. 3-5, where "track gate" refers to the estimated spatial extent of the target. The LWIR and MWIR planes of the dual-band tracked target appearance $z_{k}$ in (4) (without the noise $\mathbf{n}_{k}$ ) are shown superimposed on the upper corners of the bottom image in each group of three.

The dual-band DBSIR algorithm delivered the best performance overall, achieving the smallest RMS tracking error in nine of the 17 test sequences and maintaining track lock in all cases. The number of DBSIR appearance model updates is given in the rightmost column of Table II. Against the heavily cluttered metropolitan SB sequences, DBSIR was the best performer in every case tested. Against the $\mathrm{BC}$ sequences acquired on a rural highway just outside Bishop, CA (see Figs. 3(a), 5(a)), DBSIR performed best in four out of the seven cases tested and was within 1.5 pixels of the best performer in all other cases. Moreover, DBSIR maintained track lock against all of the sequences. Against the airport sequences SA1 and SA2 (see Fig. 3(b)), DBSIR was not
TABLE II

RMS ERROR IN TRACKED CENTROID FOR 17 TEST TARGETS. LWSIR AND MWSIR ARE THE SINGLE-BAND PARTICLE FILTERS OPERATING INDEPENDENTLY IN THE LWIR AND MWIR BANDS. DBSIR IS THE

PARTICLE FILTER OPERATING JOINTLY IN BOTH BANDS. ERROR STATISTICS ARE AVERAGED OVER 40 RUNS FOR EACH TARGET. AN ERROR OF $\infty$ MEANS THAT THE TRACK WAS LOST. THE LAST COLUMN GIVES THE NUMBER OF DBSIR APPEARANCE MODEL UPDATES.

\begin{tabular}{|c|c||c|c|c||c|}
\cline { 3 - 6 } \multicolumn{2}{l||}{} & \multicolumn{3}{c|}{ RMS Tracking Error (pix) } & DBSIR \\
Target & \# frames & LWSIR & MWSIR & DBSIR & updates \\
\hline \hline BC1 & 180 & 1.5951 & $\mathbf{1 . 5 0 0 2}$ & 2.8498 & 1 \\
BC2 & 300 & 1.2963 & $\mathbf{0 . 8 6 2 9}$ & 2.2879 & 1 \\
BC3 & 115 & $\infty$ & $\infty$ & $\mathbf{2 . 4 6 8 2}$ & 2 \\
BC4 & 160 & $\infty$ & $\infty$ & $\mathbf{2 . 3 2 3 4}$ & 2 \\
BC5 & 275 & 2.0505 & 2.0938 & $\mathbf{1 . 5 4 0 2}$ & 2 \\
BC6 & 130 & $\infty$ & $\mathbf{1 . 4 0 9 3}$ & 2.4063 & 5 \\
BC7 & 380 & 2.6601 & 1.2119 & $\mathbf{1 . 1 8 2 2}$ & 0 \\
\hline SA1 & 72 & $\mathbf{1 . 1 8 7 7}$ & 1.3611 & 1.2325 & 0 \\
SA2 & 200 & 1.9390 & $\mathbf{1 . 7 0 3 1}$ & 1.8870 & 0 \\
\hline SB1 & 80 & 2.4600 & 2.1293 & $\mathbf{1 . 7 4 2 0}$ & 2 \\
SB2 & 89 & 1.3616 & 1.3287 & $\mathbf{1 . 2 2 7 8}$ & 1 \\
SB3 & 178 & 2.0687 & 1.6663 & $\mathbf{1 . 5 1 2 7}$ & 1 \\
SB4 & 213 & 1.6194 & 1.7727 & $\mathbf{1 . 5 5 6 1}$ & 2 \\
\hline VN1 & 200 & 3.7645 & 3.7471 & $\mathbf{3 . 4 3 2 8}$ & 3 \\
VN2 & 190 & $\mathbf{5 . 0 0 7 7}$ & 5.0800 & 5.3750 & 4 \\
VN3 & 240 & $\mathbf{3 . 2 9 7 4}$ & 5.0173 & 6.2880 & 3 \\
VN4 & 300 & 1.9919 & $\mathbf{1 . 6 8 8 1}$ & 1.8732 & 1 \\
\hline
\end{tabular}

the best performer but delivered RMS tracking errors below two pixels in both of the cases tested. Finally, against the night-time grocery store sequences VN1-VN4 (see Figs. 4, 5(b)), DBSIR was best only against the pedestrian in VN1. However, it performed within one pixel of the best performer against VN2 and VN4. Although the RMS centroid error was nearly 6.3 pixels against $\mathrm{VN} 3$, the target in this case was large, spanning more than $100 \times 100$ pixels by the end of the sequence.

In six of the test sequences - $\mathrm{BC} 1, \mathrm{BC} 2, \mathrm{SA} 1, \mathrm{SB} 3$, SB4 (Fig. 3(d)), and VN4 (Fig. 4(b)) - there was good signal-to-clutter ratio and mild target aspect changes along with mild to substantial target magnification changes. In these cases, the dual-band fusion and appearance model updates of DBSIR provided little or no advantage relative to LWSIR and MWSIR; all three algorithms tracked well and delivered RMS centroid errors within two pixels of one another. Against these sequences, MWSIR was the best performer in three cases, DBSIR was best in two cases, and LWSIR was best in one case.

Against BC3 (Fig. 3(a)), BC4, BC6, BC7, SB1, and SB2, structured clutter interfered with the LWSIR and/or MWSIR single-band trackers, causing errors in the target magnification estimation. Against $\mathrm{BC} 3$ and $\mathrm{BC} 4$, these errors were severe 

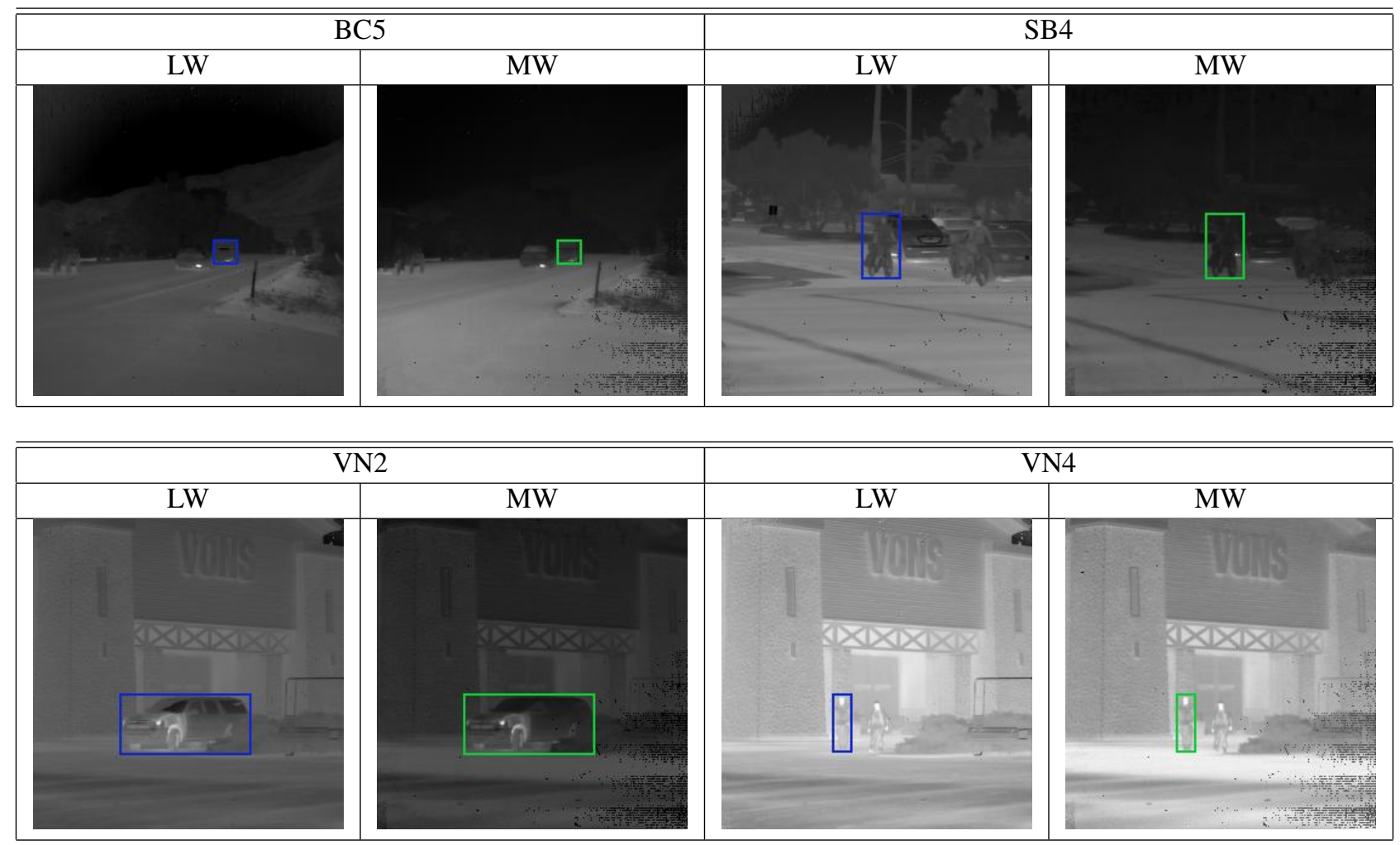

Fig. 2. Sample LWIR/MWIR frame pairs for four of the test targets. Ground truth is shown by blue and green overlayed rectangles.
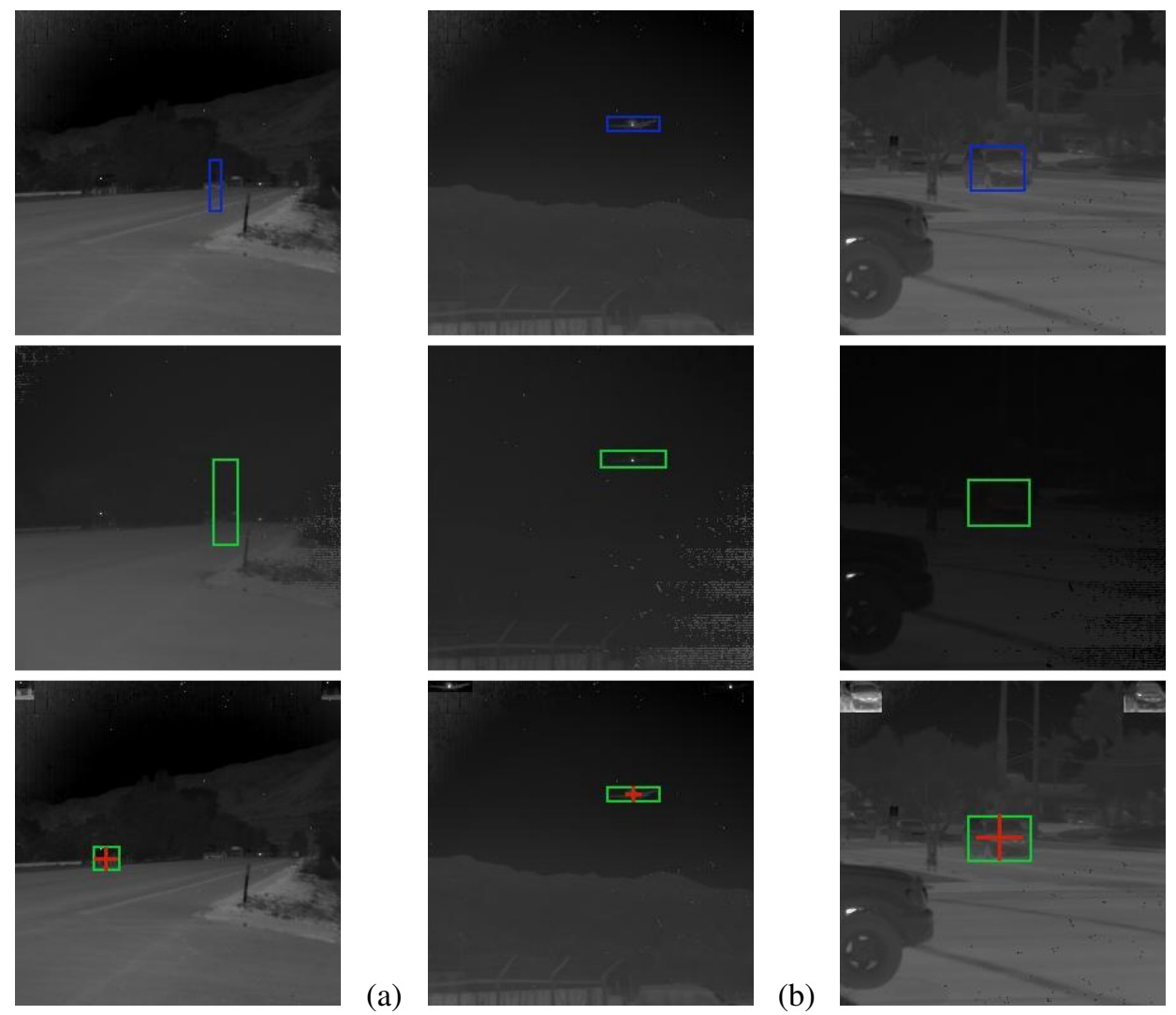

(a)
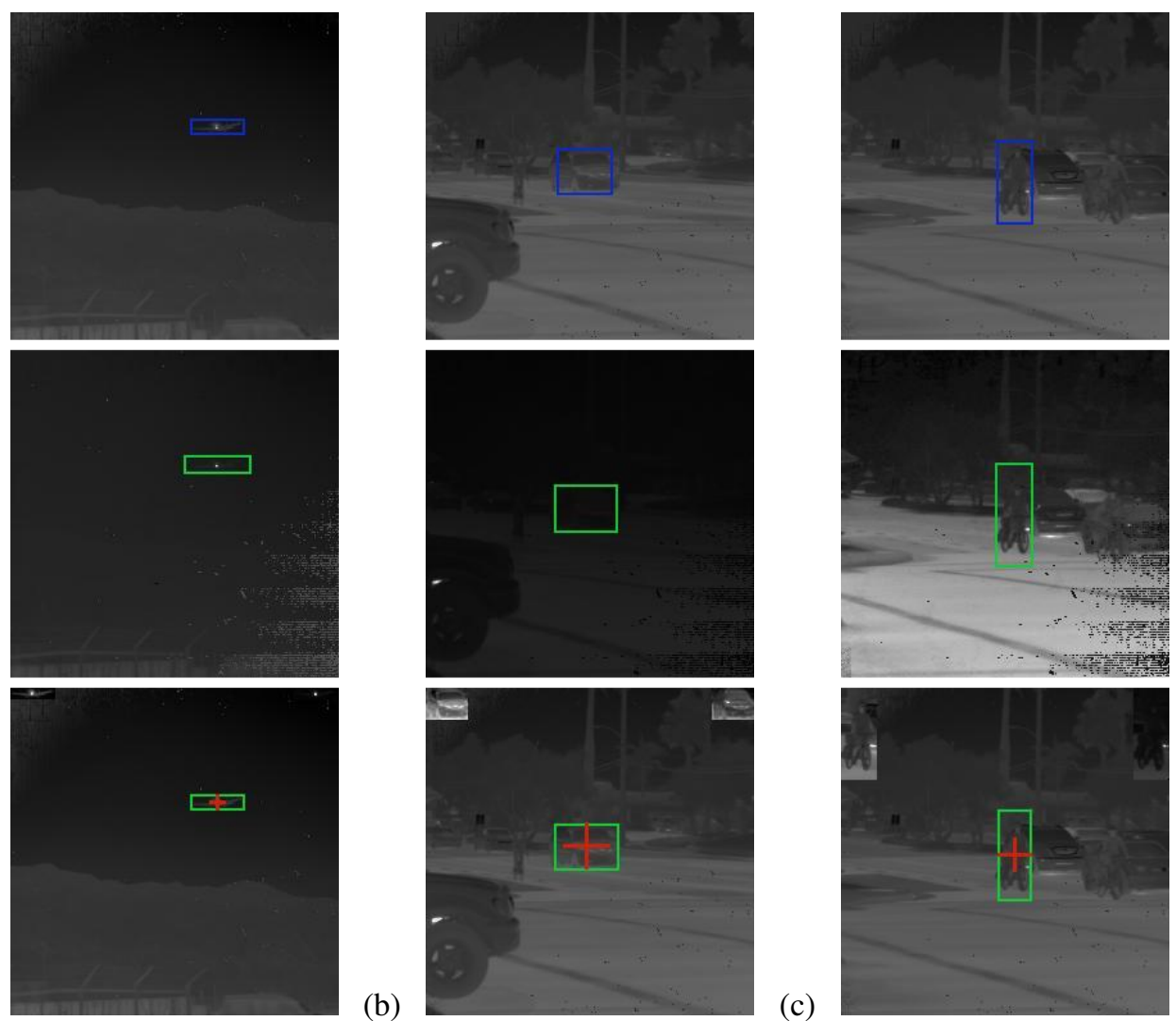

(d)

Fig. 3. Tracking examples. For each column: top - LWSIR track gate; middle - MWSIR track gate; bottom - DBSIR dual-band track gate superimposed on the LWIR frame. The LWIR and MWIR planes of the dual-band template are superimposed on the upper left/right corners of the bottom image. (a) BC3, frame 72. LWSIR has switched to an incorrect target and MWSIR is hung on clutter. (b) SA2, frame 51. (c) SB2, frame 64. (d) SB4, frame 156. 

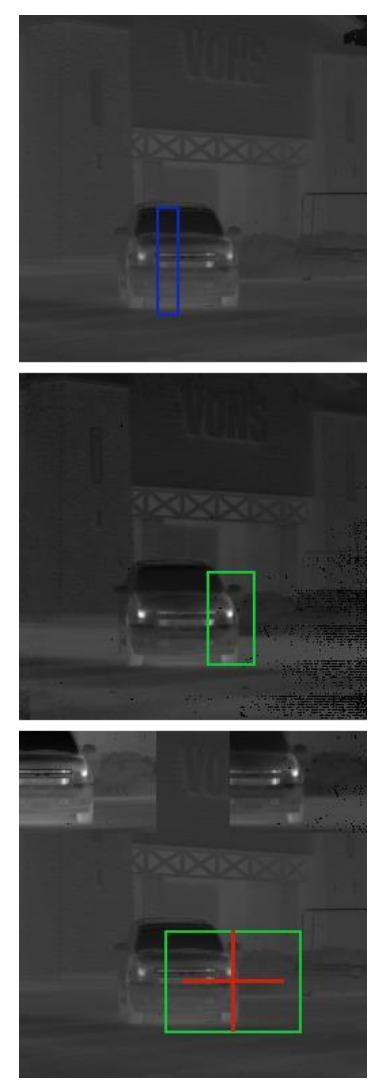

(a)
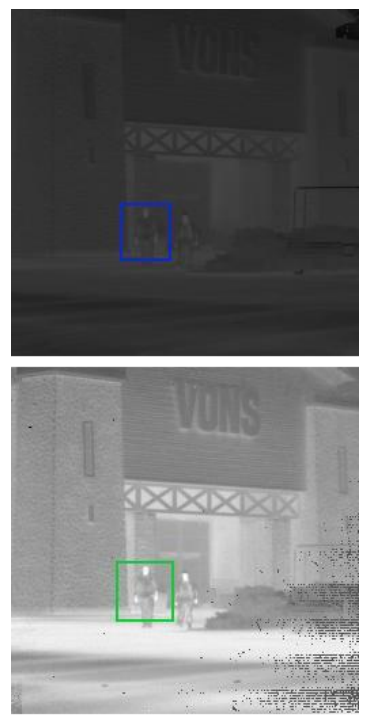

(b)

Fig. 4. VN series tracking examples. For each column: top - LWSIR track gate; middle - MWSIR track gate; bottom - DBSIR dual-band track gate superimposed on the LWIR frame. The LWIR and MWIR planes of the dualband template are superimposed on the upper left/right corners of the bottom image. (a) VN3, frame 166. (b) VN4, frame 200.

enough to result in LWSIR and MWSIR track losses. In the case of BC6, the LWSIR tracker was distracted by clutter and then locked onto a car driving in the opposite direction of the target; the ambiguities in the longwave data through this event also degraded the performance of DBSIR momentarily, resulting in an overall better performance figure for MWSIR. The advantages of dual-band fusion and appearance model updates enabled DBSIR to achieve the best performance against the remaining five sequences in this group.

On SA2, VN2, and VN3, all three trackers lost the overall target shape and locked onto small bright features. These sequences are all characterized by substantial magnification and aspect changes. However, against SA2 (Fig. 3(b)) all three algorithms ignored the aircraft fuselage and essentially tracked the much brighter engine signature only - which was relatively invariant under aspect changes, thereby nullifying the potential DBSIR advantages of dual-band fusion and appearance model updates. Similarly, against VN2 and VN3 (Fig. 4(a)), the SUV executed a turn early in both sequences after which DBSIR and MWSIR locked onto a headlamp while LWSIR locked onto the front grill. The RMS centroid error of all three algorithms was very close against SA2 and VN2. In the case of VN3, DBSIR had the largest RMS centroid error but delivered superior magnification estimates as shown in Fig. 4(a).
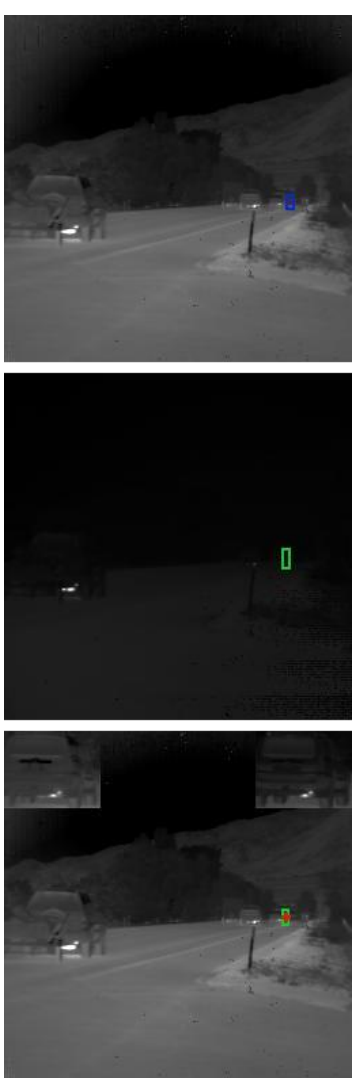

(a)
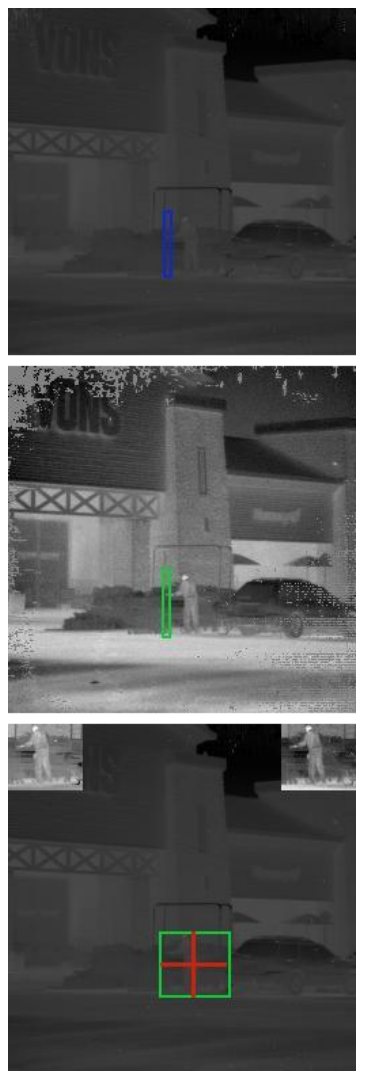

(b)

Fig. 5. Partial occlusion tracking examples. For each column: top - LWSIR track gate; middle - MWSIR track gate; bottom - DBSIR dual-band track gate superimposed on the LWIR frame. The LWIR and MWIR planes of the dual-band template are superimposed on the upper left/right corners of the bottom image. (a) BC5, frame 236. There is a partial occlusion from the vehicle behind the target. (b) VN1, frame 196. Around frame 175, the man passed behind the poles and car to the right of him. Here near the end of the sequence, an appearance model update has enabled DBSIR to recover while LWSIR and MWSIR are still locking onto other poles.

The two partial occlusion examples $\mathrm{BC} 5$ and $\mathrm{VN} 1$ are shown in Fig. 5. Against BC5, LWSIR and MWSIR were compromised by an occlusion from the vehicle behind the target which was compensated by an appearance model update by DBSIR. In VN1, the pedestrian crossed behind the rightmost poles. While this degraded the performance of the single-band algorithms, DBSIR recovered with an appearance update.

\section{DISCUSSION}

The individual effects of magnification, template update, and dual-band fusion are difficult to analyze in general. Because of the limited nature of our experiments, they are not readily apparent in the RMS tracking error results of Table II. The most significant conclusion to be drawn from Table II in our opinion is that the DBSIR filter equipped with a dual-domain track consistency check and template update and performing dual-band fusion at both the pixel and track levels was able to maintain an accurate track lock against all of the sequences - and did so without an a priori stored signature library. This is in contrast to the single-band algorithms, which lost the track against some of the $\mathrm{BC}$ sequences. We also 
conclude that the combination of MWIR/LWIR pixel-level data fusion and track-level fusion for triggering appearance updates in DBSIR generally provides robustness against partial occlusions, enhanced clutter rejection, and improved target magnification estimation relative to the single-band algorithms.

The question of how to implement a good template update strategy for the single-band trackers is not entirely straightforward. Naïve approaches such as "update every frame" and fixed interval update strategies tend to eventually lock onto clutter and fail; they did not succeed very well against the 17 sequences studied here. Our future work will include applying other state-of-the-art video trackers to this data for comparison. Another limitation of this work is that none of the tested sequences provided ambient conditions such as fog, smoke, haze, and precipitation, all of which are expected to impact the two bands differently.

\section{REFERENCES}

[1] G. Ariyawansa, M. Gupen, J. Duran, J. Scheihing, T. Nelson, and M. Eismann, "Design and modeling of InAs/GaSb type II superlattice based dual-band infrared detectors," J. Applied Phys., vol. 111, pp. 1-10, 2012.

[2] S. Eker, M. Kaldirim, Y. Arslan, and C. Besikei, "Large-format voltagetunable dual-band quantum-well infrared photodetector focal plane array for third-generation thermal imagers," IEEE Electron. Device Lett., vol. 29, no. 10, pp. 1121-1123, Oct. 2008.

[3] V. Guériaux, N. de l'Isle, A. Berurier, O. Huet, A. Manissadjian, H. Facoetti, X. Marcadet, M. Carras, V. Trinité, and A. Nedelcu, "Quantum well infrared photodetectors: present and future," Optical Engr., vol. 50, no. 6, pp. 1-19, Jun. 2011.

[4] P. Abbott, L. Pillans, P. Knowles, and R. McEwen, "Advances in dualband IRFPAs made from $\mathrm{HgCdTe}$ grown by MOVPE," in Infrared Tech., Applications XXXVI, ser. Proc. SPIE, B. Andersen, G. Fulop, and P. Norton, Eds., vol. 7660, 2010, pp. 1-11.

[5] Y. Reibel, F. Chabuel, C. Vaz, D. Billon-Lanfrey, J. Baylet, O. Gravrand, P. Ballet, and G. Destefanis, "Infrared dual band detectors for next generation," in Infrared Tech., Applications XXXVII, ser. Proc. SPIE, B. Andersen, G. Fulop, and P. Norton, Eds., vol. 8012, 2011, pp. 1-13.

[6] D. Scribner, J. Schuler, P. Warren, M. Satyshur, and M. Kruer, "Infrared color vision: separating objects from backgrounds," in Infrared Detectors and Focal Plane Arrays V, ser. Proc. SPIE, vol. 3379, 1998, pp. 2-13.

[7] A. Goldberg, B. Stann, and N. Gupta, "Multispectral, hyperspectral, and three-dimensional imaging research at the U.S. Army Research Laboratory," in Proc. Int'l. Conf. Image Fusion, Cairns, Australia, Jul. 8 - 11, 2003, pp. 499-506.

[8] S. Moyer, R. Driggers, R. Vollmerhausen, M. Soel, G. Welch, and W. Rhodes, "Information differences between subbands of the mid-wave infrared spectrum," Optical Engr., vol. 42, no. 8, pp. 2296-2303, Aug. 2003.

[9] J. Jackman, M. Richardson, B. Butters, and R. Walmsley, "Countermeasure effectiveness against a man-portable air-defense system containing a two-color spinscan infrared seeker," Optical Engr., vol. 50, no. 12, pp. 1-10, Dec. 2011.

[10] A. Maini and V. Agrawal, "Test system for comprehensive evaluation of infared-guided missiles," IET Optoelectr., vol. 6, no. 5, pp. 255-262, 2012.

[11] M. de Visser, P. Schwering, J. de Groot, and E. Hendriks, "Passive ranging using an infrared search and track sensor," Optical Engr., vol. 45, no. 2, pp. 1-14, Feb. 2006.

[12] C. Quaranta and G. Balzarotti, "Probabilistic data association appliactions to data fusion," Optical Engr, vol. 47, no. 2, pp. 1-11, Feb. 2008.

[13] F. Neele, "Two-colour infrared missile warning sensors," in Airborne Intell., Surveillance, Reconn. Syst., Appl. II, ser. Proc. SPIE, S. Wyatt, Ed., vol. 5787, 2005, pp. 134-145.

[14] M. Münzberg, R. Breiter, W. Cabanski, K. Hofmann, H. Lutz, J. Wendler, J. Ziegler, R. Rehm, and M. Walther, "Dual color IR detection modules, trends and applications," in Infrared Tech., Applications XXXIII, ser. Proc. SPIE, B. Andersen, G. Fulop, and P. Norton, Eds., vol. 6542,2007 , pp. 1-8.
[15] I. Hirsh, L. Shkedy, D. Chen, N. Fishler, Y. Hagbi, A. Koifman, Y. Openhaim, I. Vaserman, M. Singer, and I. Shtrichman, "Hybrid dualcolor MWIR detector for airborne missile warning system," in Infrared Tech., Applications XXXVII, ser. Proc. SPIE, B. Andersen, G. Fulop, and P. Norton, Eds., vol. 8353, 2012, pp. 1-12.

[16] M. Tomkinson, B. Teaney, and J. Olson, "Dual band sensor fusion for urban target acquisition," in Infrared Imaging Syst.: Design, Anal., Modeling, and Testing XVI, ser. Proc. SPIE, G. Holst, Ed., vol. 5784, 2005, pp. 27-34.

[17] T. Maurer, D. Wilson, S. Smith, D. Deaver, E. Flug, and O. Nguyen, "Search and detection comparing midwave and longwave infrared," Optical Engr., vol. 48, no. 11, pp. 1-9, Nov. 2009.

[18] U. Adomeit and R. Ebert, "Improved target detection by IR dual-band image fusion," in Electro-Optical, Infrared Syst.: Tech. and Appl. VI, ser. Proc. SPIE, D. Huckridge and R. Ebert, Eds., vol. 7481, 2009, pp. $1-8$.

[19] D. Scribner, J. Schuler, P. Warren, and G. H. M. Kruer, "Image preprocessing for the infrared," in Infrared Detectors, Focal Plane Arrays VI, ser. Proc. SPIE, E. Dereniak and R. Sampson, Eds., vol. 4028, 2000, pp. 222-233.

[20] R. Moore, C. Halford, and C. Howell, "Finding a fusion metric that best reflects human observer preference," in Infrared Imaging Syst.: Design Anal., Modeling, Testing XVIII, ser. Proc. SPIE, G. Holst, Ed., vol. 6543, 2007, pp. 1-10.

[21] M. Müller, O. Schreer, and M. Sáenz, "Real-time image processing and fusion for a new high-speed dual-band infrared camera," in Infrared Imaging Syst.: Design Anal., Modeling, Testing XVIII, ser. Proc. SPIE, G. Holst, Ed., vol. 6543, 2007, pp. 1-9.

[22] U. Adomeit and R. Ebert, "IR-dual-band-camera demonstartor experimental assessment - practical applications," in Electro-Optical, Infrared Syst.: Tech., Appl. IV, ser. Proc. SPIE, D. Huckridge and R. Ebert, Eds., vol. 6737, 2007, pp. 1-11.

[23] Q. Qingwang, G. Kun, and N. Guoqiang, "Image fusion and its real-time processing in dual-band infrared night vision systems," in Int'l. Conf. Optical Instr., Tech: Optical Syst., Optoelectr. Instr, ser. Proc. SPIE, Y. Sheng, Y. Wang, and L. Zeng, Eds., vol. 7156, 2009, pp. 1-9.

[24] C. Quaranta, A. Ondini, L. Fortunato, and G. Balzarotti, "IMM techniques for dual band infrared target tracking," in Image, Signal Process. Remote Sens. X, ser. Proc. SPIE, L. Bruzzone, Ed., vol. 5573, 2004, pp. 238-249.

[25] W. Bingjian, L. Gang, B. Liping, L. Qing, and L. Shangqian, "A new small and dim targets detection and recognition algorithm based on infrared dual bands imaging system," in Int'l. Symp. Photoelectron. Detection, Imaging: Advances Infrared Imaging and Appl., ser. Proc. SPIE, J. Puschell, J. Chu, H. Gong, and J. Lu, Eds., vol. 8193, 2011, pp. $1-8$.

[26] Y.-Q. Sun, J.-W. Tian, and J. Liu, "Novel method on dual-band infrared image fusion for dim small target detection," Optical Engr., vol. 46, no. 11 , pp. $1-8$, Nov. 2007

[27] P. Dainty, J. Boyce, C. Dimitropoulos, and M. Bernhardt, "Dual-band ATR for forward-looking infrared images," in Proc. IEEE Workshop Comput. Vision Beyond Visible Spectrum: Methods, Appl., Fort Collins, CO, Jun. 21-22, 1999, pp. 23-29.

[28] D. Fay, A. Waxman, R. Ivey, N. Bomberger, and M. Chiarella, "Multisensor image fusion \& mining: learning targets across extended operating conditions," in Enhanced, Synthetic Vis., ser. Proc. SPIE, J. Verly, Ed., vol. 5424, 2004, pp. 148-162.

[29] L. Chan, S. Der, and N. Nasrabadi, "Dual-band passive infrared imagery for automatic clutter rejection," in Proc. IEEE Int'l. Conf. Image Proc., vol. 2, Vancouver, Canada, Sep. 10-13, 2000, pp. 463-466.

[30] — "Dual-band FLIR fusion for target detection," in Appl. Artificial Neural Networks Image Process. VII, ser. Proc. SPIE, N. Masrabadi and A. Katsaggelos, Eds., vol. 4668, 2002, pp. 108-118.

[31] A. Wei $\beta$, U. Adomeit, P. Chevalier, S. Landeau, P. Bijl, F. Champagnat, J. Dijk, B. Göler, S. Landini, J. Reynolds, and L. Smith, "A standard data set for performance analysis of advanced IR image processing techniques," in Infrared Imaging Syst.: Design, Anal., Modeling, Testing XXIII, ser. Proc. SPIE, G. Holst and K. Krapels, Eds., vol. 8355, 2012, pp. $1-10$.

[32] S. Arulampalam, S. Maskell, N. Gordon, and T. Clapp, "A tutorial on particle filters for online non-linear/non-Gaussian Bayesian tracking," IEEE Trans. Signal Process., vol. 50, no. 2, pp. 174-188, 2002. 